\title{
О ТОЧНОСТИ АППРОКСИМАЦИИ ДВИЖЕНИЯ МАЛОГО НЕБЕСНОГО ТЕЛА ПРОМЕЖУТОЧНЫМИ ВОЗМУЩЕННЫМИ ОРБИТАМИ, ПОСТРОЕННЫМИ ПО ТРЕМ ВЕКТОРАМ ПОЛОЖЕНИЯ И ТРЕМ НАБЛЮДЕНИЯМ
}

\author{
(C) 2016 г. В. А. Шефер ${ }^{\text {, O. В. Шефер }}{ }^{2}$ \\ ${ }^{1}$ Научно-исследовательский институт прикладной математики \\ и механики Томского государственного университета, Томск, Россия \\ ${ }^{2}$ Институт кибернетики, Томский политехнический университет, Томск, Россия \\ e-mail: shefer@niipmm.tsu.ru \\ Поступила в редакцию 01.06.2015 г.
}

\begin{abstract}
Рассматриваются введенные ранее первым автором промежуточные возмущенные орбиты, которые строятся по трем векторам положения и трем измерениям угловых координат малого небесного тела. При малом опорном интервале времени, охватывающем измерения, эти орбиты по степени аппроксимации реального движения близки к орбите с касанием четвертого порядка. Чем меньше опорный временной интервал, тем лучше выполняется это свойство. Формулируются законы изменения погрешностей методов построения промежуточных орбит в зависимости от длины опорного интервала времени. Согласно этим законам скорость сходимости методов к точному решению при сокращении опорного интервала времени в общем случае на три порядка выше, чем в традиционных методах, основанных на использовании невозмущенной кеплеровской орбиты. Рассматриваемые орбиты являются одними из наиболее точных среди орбит своего класса, определяемого порядком касания. Теоретические результаты исследования подтверждаются численными экспериментами на примерах определения орбиты астероида Апофис.
\end{abstract}

\begin{abstract}
Ключевые слова: промежуточная возмущенная орбита, сверхоскулирующая орбита, определение первоначальной орбиты, метод Херрика-Гиббса.
\end{abstract}

DOI: $10.7868 / \mathrm{S} 0320930 \mathrm{X} 16030063$

\section{ВВЕДЕНИЕ}

В статьях (Шефер, 2007; 2010) на основе развитой ранее теории сверхоскулирующих орбит с касанием четвертого порядка (Шефер, 2006) предложены методы построения промежуточных возмущенных орбит малых небесных тел по небольшому числу граничных условий. Это метод вычисления промежуточной орбиты по трем векторам положения в три момента времени и метод определения промежуточной орбиты по трем положениям на небесной сфере и соответствующим им моментам времени. Методы позволяют учитывать основную часть возмущений, оказывающих влияние на движение исследуемого тела. Это удается осуществить благодаря тому, что движение по промежуточным орбитам представляется комбинацией из двух движений: движения фиктивного притягивающего центра, гравитационный параметр которого изменяется по первому закону Мещерского вариации массы, и движения относительно фиктивного центра. Первое движение в общем случае происходит по параболе с постоянным ускорением, а второе описывается уравнениями задачи
Гюльдена-Мещерского. Решения для промежуточных орбит могут быть представлены в замкнутой аналитической форме.

В данной работе исследуется вопрос о точности аппроксимации возмущенного движения предложенными в (Шефер, 2007; 2010; ниже S07 и S10 соответственно) промежуточными орбитами. Формулируются два утверждения о законах изменения погрешностей методов построения промежуточных орбит. Справедливость этих законов подтверждается результатами численных экспериментов.

Заметим, что данная работа сходна по тематике с работой, выполненной нами ранее (Шефер В., Шефер О., 2015; ниже SS15). Основное отличие между ними состоит в том, что предметом исследования в предлагаемой работе являются промежуточные орбиты, которые по степени аппроксимации реального движения близки к сверхоскулирующей орбите, на порядок более точной, чем в случаях, рассмотренных в SS15. Мы сохраняем здесь, если это не оговаривается отдельно, введенные в SS15 обозначения. 


\section{ПРОМЕЖУТОЧНЫЕ ВОЗМУЩЕННЫЕ ОРБИТЫ. ЗАКОНЫ ИЗМЕНЕНИЯ ПОГРЕШНОСТЕЙ МЕТОДОВ ИХ ПОСТРОЕНИЯ}

Рассмотрим движение малого тела (астероид, комета, крупный метеороид, космический аппарат) под действием ньютоновского притяжения системы точечных масс (Солнце, планеты, спутники планет), из которых одна масса принимается за центральную (основное тело). Уравнения движения запишем в форме (SS15)

$$
\ddot{\mathbf{x}}=-\frac{K}{r^{3}} \mathbf{x}+\mathbf{F} \equiv \mathbf{G},
$$

где $\mathbf{x}-$ вектор положения малого тела, $r=|\mathbf{x}|$, $K=f M=$ const $(f-$ гравитационная постоянная, $M$ - масса основного тела), $\mathbf{F}$ - вектор возмущающего ускорения. Пусть в начальный момент $t=t_{0}$ известны векторы положения и скорости малого тела

$$
\mathbf{x}\left(t_{0}\right)=\mathbf{x}_{0}, \quad \dot{\mathbf{x}}\left(t_{0}\right)=\dot{\mathbf{x}}_{0} .
$$

Движение, описываемое уравнениями (1) и условиями (2), назовем реальным.

Будем считать, что в интервале времени, в котором изучается движение малого тела, правые части (1) обладают производными по $t$ любого порядка.

Введенные для реального движения величины, обозначенные ниже символом *, сохраняют свой смысл, но относятся к промежуточному движению. Верхний индекс в скобках обозначает порядок производной по $t$.

Пусть даны три различных положения малого тела на реальной траектории в моменты времени $t_{1}, t_{2}, t_{3}\left(t_{1}<t_{2}<t_{3}\right)$, определяемые векторами

$$
\mathbf{x}\left(t_{1}\right)=\mathbf{x}_{1}, \quad \mathbf{x}\left(t_{2}\right)=\mathbf{x}_{2}, \quad \mathbf{x}\left(t_{3}\right)=\mathbf{x}_{3} .
$$

Введем обозначения

$$
t_{12}=t_{2}-t_{1}, t_{23}=t_{3}-t_{2}, t_{13}=t_{3}-t_{1} .
$$

Будем рассматривать интервалы (4) как малые величины первого порядка относительно максимальной из длин этих интервалов $t_{13}$.

B S07 изложен метод построения промежуточной возмущенной орбиты, на которой положения и ускорения в моменты $t_{1}, t_{2}$ и $t_{3}$ совпадают с положениями, задаваемыми векторами (3), и суммарными ускорениями $\mathbf{G}_{1}, \mathbf{G}_{2}$ и $\mathbf{G}_{3}$ соответственно. Для краткости будем ссылаться на нее как на орбиту $S_{\text {pos }}$.

\section{Сформулируем утверждение:}

УТВЕРЖДЕНИЕ 1. Если решения задачи Коши (1), (2) являются функциями, аналитическими в открытом интервале, содержащем замкнутый интервал $\left[t_{1}, t_{3}\right]$, то промежуточная орбита $S_{\text {pos }}$ в пределе при $\left(t_{3}-t_{1}\right) \rightarrow 0$ является орбитой с каса- нием четвертого порядка к траектории движения, описываемого уравнениями (1) и начальными условиями (2). При этом $\mathbf{x} *\left(t_{i}\right)=\mathbf{x}\left(t_{i}\right), \ddot{\mathbf{x}}^{*}\left(t_{i}\right)=\mathbf{G}\left(t_{i}\right)$, а величины $\left|\dot{\mathbf{x}}^{*}\left(t_{i}\right)-\dot{\mathbf{x}}\left(t_{i}\right)\right|,\left|\mathbf{x}^{*(3)}\left(t_{i}\right)-\dot{\mathbf{G}}\left(t_{i}\right)\right|$ и $\left|\mathbf{x}^{*(4)}\left(t_{i}\right)-\ddot{\mathbf{G}}\left(t_{i}\right)\right|$ уменьшаются как $\left(t_{3}-t_{1}\right)^{4},\left(t_{3}-t_{1}\right)^{2}$ и $\left(t_{3}-t_{1}\right)^{m}$ соответственно; $i=1,2,3 ; m=1$. В частном случае, когда $i=2$ и $t_{12}=t_{23}, m=2$.

Векторы $\mathbf{x}^{*(k)}\left(t_{i}\right)(k=2,3,4)$ определяются с помощью формул (22)-(24) из (Шефер, 2006).

Пусть для каждого из трех моментов времени $t_{1}^{\circ}, t_{2}^{\circ}, t_{3}^{\circ}\left(t_{1}^{\circ}<t_{2}^{\circ}<t_{3}^{\circ}\right)$ имеется пара наблюденных угловых координат. Угловые координаты, полученные в момент $t_{i}^{\circ}$, можно представить в виде единичного вектора $\mathbf{L}_{i}$. Предположим, что наблюденные величины не содержат ошибок и $\left[\left(\mathbf{L}_{1} \times \mathbf{L}_{2}\right) \cdot \mathbf{L}_{3}\right] \neq 0$.

Справедливы равенства

$$
\mathbf{x}_{i}=\rho_{i} \mathbf{L}_{i}-\mathbf{S}_{i}, \quad t_{i}=t_{i}^{\circ}-\frac{1}{c} \rho_{i} ; \quad i=1,2,3 .
$$

Здесь $\mathbf{x}_{i}-$ вектор положения малого тела в момент $t_{i} ; \rho_{i}$ - дальность (расстояние от точки наблюдения до малого тела); $\mathbf{S}_{i}-$ вектор положения основного тела относительно точки наблюдения, определенный в момент $t_{i}^{\circ} ; c-$ скорость света.

B S10 решена задача построения промежуточной возмущенной орбиты, точно удовлетворяющей трем указанным парам угловых измерений. Назовем ее орбитой $S_{\text {obs }}$.

Для $S_{\text {obs }}$ равенства (5) перепишутся соответственно в виде

$$
\mathbf{x}_{i}^{*}=\rho_{i}^{*} \mathbf{L}_{i}-\mathbf{S}_{i}, \quad t_{i}^{*}=t_{i}^{\circ}-\frac{1}{c} \rho_{i}^{*} ; \quad i=1,2,3 .
$$

Следовательно, в отличие от движения по $S_{\text {pos }}$, все величины, связанные с движением по $S_{\mathrm{obs}}$, соотносятся с временем $t^{*}$.

\section{Запишем следующее утверждение:}

УТВЕРЖДЕНИЕ 2. Если решения задачи Коши (1), (2) являются функциями, аналитическими в открытом интервале, содержащем замкнутый интервал $\left[t_{1}, t_{3}\right]$, то промежуточная орбита $S_{\text {obs }}$ в пределе при $\left(t_{3}-t_{1}\right) \rightarrow 0$ является орбитой с касанием четвертого порядка к траектории движения, описываемого уравнениями (1) и начальными условиями (2). При этом величины $\left|t_{i}^{*}-t_{i}\right|$, $\left|\mathbf{x}^{*}\left(t_{i}^{*}\right)-\mathbf{x}\left(t_{i}\right)\right|,\left|\dot{\mathbf{x}}^{*}\left(t_{i}^{*}\right)-\dot{\mathbf{x}}\left(t_{i}\right)\right|$ и $\left|\dot{\mathbf{x}}^{*}\left(t_{i}^{*}\right)-\mathbf{G}\left(t_{i}\right)\right|$ уменьшаются как $\left(t_{3}-t_{1}\right)^{n},\left|\mathbf{x}^{*(3)}\left(t_{i}^{*}\right)-\dot{\mathbf{G}}\left(t_{i}\right)\right|-$ как $\left(t_{3}-t_{1}\right)^{2}$, а 
$\left|\mathbf{x}^{*(4)}\left(t_{i}^{*}\right)-\ddot{\mathbf{G}}\left(t_{i}\right)\right|-$ как $\left(t_{3}-t_{1}\right)^{m}$. Здесь $n=3$ при $t_{12} \neq t_{23}, n=4$ при $t_{12}=t_{23} ; i=1,2,3 ; m=1$. В частном случае, когда $i=2$ и $t_{12}=t_{23}, m=2$.

Векторы $\mathbf{x}^{*(k)}\left(t_{i}^{*}\right)(k=2,3,4)$ находятся с помощью формул (22)-(24) из (Шефер, 2006), в которых $t_{i}$ необходимо заменить на $t_{i}^{*}$.

В связи с ограничениями, накладываемыми на объем журнальной статьи, доказательства приведенных утверждений опускаются.

Замечание 1. Мы предполагаем, что вектор $\mathbf{F}$ определяется ньютоновским притяжением возмущающих точечных масс. Однако применение методов построения орбит $S_{\mathrm{pos}}$ и $S_{\mathrm{obs}}$ возможно и в случае возмущающих сил более общего вида (см. S07).

Замечание 2. Утверждение 1 распространяется на любые орбиты, аппроксимирующие движение (1), (2) аналитическими функциями и удовлетворяющие граничным условиям (22) и (23) из S07. Подробности более широкого подхода к построению промежуточных орбит с касанием четвертого порядка можно найти в (Шефер, 2006).

Замечание 3. В S07 получена формула Херрика-Гиббса для вычисления вектора скорости в средний момент времени по значениям векторов положения в три различные момента времени, обобщенная на случай возмущенного движения. Отклонения вектора скорости, найденного по этой формуле, от реального вектора скорости $\dot{\mathbf{x}}_{2}$ при малом интервале $\left(t_{3}-t_{1}\right)$ прямо пропорциональны этому интервалу, возведенному в четвертую степень. Обозначим орбиту, определенную с помощью указанной формулы Херрика-Гиббса, через $H_{\text {pos }}$.

Замечание 4. В S10 предложены формулы обобщенного метода Херрика-Гиббса вычисления промежуточной орбиты по трем наблюдениям. Этот метод представляет собой развитие метода, предложенного Herrick (1952). По точности аппроксимации реального движения его можно рассматривать как альтернативу методу построения орбиты $S_{\text {obs }}$. Ниже будем говорить об орбите, определенной с помощью обобщенного метода Херрика-Гиббса (S10), как об орбите $H_{\text {obs }}$.

Замечание 5. Орбита $H_{\mathrm{obs}}$, согласно ее построению, определяет невозмущенную кеплеровскую орбиту с той же точностью, что и возмущенную. Что же касается орбиты $S_{\text {obs, }}$, то при отсутствии возмущений она задает кеплеровскую орбиту точно. Это означает, что отклонения параметров $S_{\text {obs }}$ от параметров реальной орбиты в общем случае пропорциональны не только величине $\left(t_{3}-t_{1}\right)^{3}$, но и малому возмущающему коэффициенту. Поэтому на практике при использовании $S_{\text {obs }}$ в общем случае следует ожидать уменьшения абсолютных значений ошибок вычисления орбиты в сравнении с использованием $H_{\mathrm{obs}}$. В следующем разделе мы найдем этому подтверждение.

Замечание 6. Замечание 7 из SS15 распространяется и на орбиты $H_{\text {obs }}$ и $S_{\text {obs }}$.

\section{ЧИСЛЕННЫЕ ЭКСПЕРИМЕНТЫ}

С целью численной проверки полученных теоретических результатов нами были разработаны программы, реализующие методы построения орбит $S_{\text {pos }}, S_{\text {obs }}, H_{\text {pos }}$ и $H_{\text {obs }}$ при машинных расчетах.

Ранее (S07, S10) на примерах нахождения орбит малых планет Адеона (145 Adeona), Икар (1566 Icarus) и Тоутатис (4179 Toutatis) были получены численные оценки погрешностей рассматриваемых методов построения орбит в зависимости от длины опорного интервала времени. Эти результаты показывают, что сформулированные в предыдущем разделе утверждения, а также Замечание 6, находят практическое подтверждение.

Здесь, с целью более обстоятельного анализа, рассматриваются еще несколько примеров, связанных с определением орбиты астероида Апофис (99942 Apophis). Постановка соответствующих численных экспериментов подробно описана в SS15. Использовались тройки положений астероида в эпохи, равноотстоящие, когда $t_{12}=t_{23}$, и неравноотстоящие, когда $t_{12}: t_{23}=2: 1$, относительно средней эпохи $t_{2}$, указанной в SS15. Результаты вычислений приводятся в табл. 1-4. В таблицах приняты обозначения: $\vartheta_{j}-$ значение истинной аномалии в момент $t_{j}(j=1,3) ; \Delta t_{2}=t_{2}^{*}-t_{2}, \Delta r_{2}=\left|\mathbf{x}_{2}^{*}-\mathbf{x}_{2}\right|, \Delta v_{2}=$ $=\left|\dot{\mathbf{x}}_{2}^{*}-\dot{\mathbf{x}}_{2}\right|, \Delta w_{2}=\left|\ddot{\mathbf{x}}_{2}^{*}-\mathbf{G}_{2}\right|, \Delta w_{2}^{\prime}=\left|\mathbf{x}_{2}^{*(3)}-\dot{\mathbf{G}}_{2}\right|, \Delta w_{2}^{\prime \prime}=$ $=\left|\mathbf{x}_{2}^{*(4)}-\ddot{\mathbf{G}}_{2}\right|-$ ошибки в моменте времени, векторах положения, скорости, ускорения и производных 1-го и 2-го порядков от вектора ускорения, соответствующие эпохе $t_{2} ; N_{t}, N_{r}, N_{\mathrm{v}}, N_{w}, N_{w^{\prime}}, N_{w^{\prime \prime}}-$ отношения значений ошибок в моменте времени, векторах положения, скорости, ускорения и производных 1-го и 2-го порядков от вектора ускорения, приведенных в данной строке таблицы, к значениям этих же ошибок, приведенных в предыдущей строке таблицы. Здесь $\mathbf{x}_{2}, \dot{\mathbf{x}}_{2}, \mathbf{G}_{2}, \dot{\mathbf{G}}_{2}$, $\ddot{\mathbf{G}}_{2}$ - векторы положения, скорости, ускорения и производных 1-го и 2-го порядков от вектора ускорения на номинальной траектории в момент $t_{2}$. Векторы $\mathbf{x}_{2}^{*}, \dot{\mathbf{x}}_{2}^{*}, \ddot{\mathbf{x}}_{2}^{*}, \mathbf{x}_{2}^{*(3)}, \mathbf{x}_{2}^{*(4)}$ на построенной орбите вычислены на момент $t_{2}^{*}$ (для орбит $H_{\mathrm{pos}}$ и $\left.S_{\text {роs }}: t_{2}^{*}=t_{2}, \mathbf{x}_{2}^{*}=\mathbf{x}_{2}, \ddot{\mathbf{x}}_{2}^{*}=\mathbf{G}_{2}\right)$. Значения ошибок даются в виде $n . m \mathrm{D}-k$, что означает десятичное 
Таблица 1. Погрешности метода определения орбиты $H_{\mathrm{pos}}$ на примерах построения орбиты Апофиса по трем гелиоцентрическим векторам положения

\begin{tabular}{|c|c|c|c|c|c|c|c|c|c|}
\hline \multicolumn{5}{|c|}{$H_{\mathrm{pos}}($ равноотстоящие моменты времени) } & \multicolumn{5}{|c|}{$H_{\text {pos }}$ (неравноотстоящие моменты времени) } \\
\hline $\begin{array}{c}t_{3}-t_{1}, \\
\text { сут }\end{array}$ & $\begin{array}{c}\Delta v_{2}, \\
\text { a. e./cyт }\end{array}$ & $\begin{array}{c}\Delta w_{2}, \\
\text { a. e./cyT }\end{array}$ & $\begin{array}{c}\Delta w_{2}^{\prime}, \\
\text { a. e. } / \text { сyт }^{3}\end{array}$ & $\begin{array}{c}\Delta w_{2}^{\prime \prime}, \\
\text { a. e./cyт }\end{array}$ & $\begin{array}{c}t_{3}-t_{1}, \\
\text { сут }\end{array}$ & $\begin{array}{c}\Delta v_{2}, \\
\text { a. e./cyт }\end{array}$ & $\begin{array}{c}\Delta w_{2}, \\
\text { a. e. } / \mathrm{cyT}^{2}\end{array}$ & $\begin{array}{c}\Delta w_{2}^{\prime}, \\
\text { a. e. } / \text { cyт }^{3}\end{array}$ & $\begin{array}{c}\Delta w_{2}^{\prime \prime}, \\
\text { a. e./cyт }\end{array}$ \\
\hline 0.125 & $1.8 \mathrm{D}-14$ & 0.0 & $2.6 \mathrm{D}-12$ & $5.9 \mathrm{D}-14$ & 0.09375 & $1.0 \mathrm{D}-13$ & 0.0 & $1.3 \mathrm{D}-12$ & $4.2 \mathrm{D}-11$ \\
\hline 0.25 & $1.6 \mathrm{D}-14$ & 0.0 & $1.1 \mathrm{D}-11$ & $2.4 \mathrm{D}-13$ & 0.1875 & $3.4 \mathrm{D}-14$ & 0.0 & $5.3 \mathrm{D}-12$ & $8.5 \mathrm{D}-11$ \\
\hline 0.5 & $3.0 \mathrm{D}-13$ & 0.0 & $4.2 \mathrm{D}-11$ & $9.4 \mathrm{D}-13$ & 0.375 & $9.3 \mathrm{D}-14$ & 0.0 & $2.1 \mathrm{D}-11$ & $1.7 \mathrm{D}-10$ \\
\hline 1 & $4.9 \mathrm{D}-12$ & 0.0 & $1.7 \mathrm{D}-10$ & $3.8 \mathrm{D}-12$ & 0.75 & $1.4 \mathrm{D}-12$ & 0.0 & $8.5 \mathrm{D}-11$ & $3.4 \mathrm{D}-10$ \\
\hline 2 & $7.9 \mathrm{D}-11$ & 0.0 & $6.8 \mathrm{D}-10$ & $1.5 \mathrm{D}-11$ & 1.5 & $2.3 \mathrm{D}-11$ & 0.0 & $3.4 \mathrm{D}-10$ & $6.9 \mathrm{D}-10$ \\
\hline 4 & $1.3 \mathrm{D}-9$ & 0.0 & $2.7 \mathrm{D}-9$ & $6.0 \mathrm{D}-11$ & 3 & $3.7 \mathrm{D}-10$ & 0.0 & $1.4 \mathrm{D}-9$ & $1.4 \mathrm{D}-9$ \\
\hline 8 & $2.0 \mathrm{D}-8$ & 0.0 & $1.1 \mathrm{D}-8$ & $2.4 \mathrm{D}-10$ & 6 & $5.9 \mathrm{D}-9$ & 0.0 & $5.5 \mathrm{D}-9$ & $2.9 \mathrm{D}-9$ \\
\hline 16 & $3.2 \mathrm{D}-7$ & 0.0 & 4.3D-8 & $9.6 \mathrm{D}-10$ & 12 & $9.8 \mathrm{D}-8$ & 0.0 & $2.2 \mathrm{D}-8$ & $6.0 \mathrm{D}-9$ \\
\hline 32 & $5.2 \mathrm{D}-6$ & 0.0 & $1.7 \mathrm{D}-7$ & $3.8 \mathrm{D}-9$ & 24 & $1.7 \mathrm{D}-6$ & 0.0 & $9.3 \mathrm{D}-8$ & $1.3 \mathrm{D}-8$ \\
\hline 64 & $8.5 \mathrm{D}-5$ & 0.0 & $7.1 \mathrm{D}-7$ & $1.5 \mathrm{D}-8$ & 48 & $2.9 \mathrm{D}-5$ & 0.0 & $4.0 \mathrm{D}-7$ & $3.2 \mathrm{D}-8$ \\
\hline
\end{tabular}

Таблица 2. Погрешности метода определения орбиты $S_{\mathrm{pos}}$ на примерах построения орбиты Апофиса по трем гелиоцентрическим векторам положения

\begin{tabular}{|c|c|c|c|c|c|c|c|c|}
\hline \multicolumn{9}{|c|}{$S_{\text {pos }}$ (равноотстоящие моменты времени) } \\
\hline $\begin{array}{c}t_{3}-t_{1}, \\
\text { сут }\end{array}$ & $\begin{array}{c}\vartheta_{3}-\vartheta_{1}, \\
\text { град }\end{array}$ & $\begin{array}{c}\Delta V_{2}, \\
\text { a. e./cyt }\end{array}$ & $N_{\mathrm{V}}$ & $\begin{array}{c}\Delta w_{2}, \\
\text { a. e. } / \text { cyT }^{2}\end{array}$ & $\begin{array}{c}\Delta w_{2}^{\prime}, \\
\text { a. e. } / \mathrm{cyt}^{3}\end{array}$ & $N_{w^{\prime}}$ & $\begin{array}{c}\Delta w_{2}^{\prime \prime} \\
\text { a. e./сут }\end{array}$ & $N_{w^{\prime \prime}}$ \\
\hline 0.125 & 0.126 & $3.3 \mathrm{D}-14$ & & 0.0 & $3.7 \mathrm{D}-14$ & & $6.4 \mathrm{D}-15$ & \\
\hline 0.25 & 0.252 & $1.8 \mathrm{D}-14$ & - & 0.0 & $1.5 \mathrm{D}-13$ & 4.0 & $2.6 \mathrm{D}-14$ & 4.0 \\
\hline 0.5 & 0.504 & $3.6 \mathrm{D}-15$ & - & 0.0 & $5.8 \mathrm{D}-13$ & 4.0 & $1.0 \mathrm{D}-13$ & 4.0 \\
\hline 1 & 1.008 & $7.1 \mathrm{D}-14$ & 19.6 & 0.0 & $2.3 \mathrm{D}-12$ & 4.0 & $4.1 \mathrm{D}-13$ & 4.0 \\
\hline 2 & 2.017 & $1.1 \mathrm{D}-12$ & 15.3 & 0.0 & $9.3 \mathrm{D}-12$ & 4.0 & $1.6 \mathrm{D}-12$ & 4.0 \\
\hline 4 & 4.034 & $1.7 \mathrm{D}-11$ & 15.8 & 0.0 & $3.7 \mathrm{D}-11$ & 4.0 & $6.5 \mathrm{D}-12$ & 4.0 \\
\hline 8 & 8.069 & $2.6 \mathrm{D}-10$ & 15.3 & 0.0 & $1.4 \mathrm{D}-10$ & 3.9 & $2.5 \mathrm{D}-11$ & 3.9 \\
\hline 16 & 16.154 & $3.7 \mathrm{D}-9$ & 13.9 & 0.0 & $5.1 \mathrm{D}-10$ & 3.6 & $9.1 \mathrm{D}-11$ & 3.6 \\
\hline 32 & 32.428 & $3.7 \mathrm{D}-8$ & 10.0 & 0.0 & $1.4 \mathrm{D}-9$ & 2.7 & $2.6 \mathrm{D}-10$ & 2.8 \\
\hline 64 & 65.790 & $2.0 \mathrm{D}-7$ & 5.4 & 0.0 & 2.4D-9 & 1.7 & $4.7 \mathrm{D}-10$ & 1.8 \\
\hline \multicolumn{9}{|c|}{$S_{\text {pos }}$ (неравноотстоящие моменты времени) } \\
\hline $\begin{array}{c}t_{3}-t_{1}, \\
\text { сут }\end{array}$ & $\begin{array}{c}\vartheta_{3}-\vartheta_{1}, \\
\text { град }\end{array}$ & $\begin{array}{c}\Delta v_{2}, \\
\text { a. e./cyt }\end{array}$ & $N_{\mathrm{V}}$ & $\begin{array}{c}\Delta w_{2}, \\
\text { a. е./cyт }{ }^{2}\end{array}$ & $\begin{array}{c}\Delta w_{2}^{\prime}, \\
\text { a. e./cyT }{ }^{3}\end{array}$ & $N_{w^{\prime}}$ & $\begin{array}{c}\Delta w_{2}^{\prime \prime}, \\
\text { a. e./cyт }\end{array}$ & $N_{w^{\prime \prime}}$ \\
\hline 0.09375 & 0.095 & $1.6 \mathrm{D}-13$ & & 0.0 & $1.8 \mathrm{D}-14$ & & $5.8 \mathrm{D}-13$ & \\
\hline 0.1875 & 0.189 & $3.2 \mathrm{D}-14$ & - & 0.0 & $7.3 \mathrm{D}-14$ & 4.0 & $1.2 \mathrm{D}-12$ & 2.0 \\
\hline 0.375 & 0.378 & $1.8 \mathrm{D}-14$ & - & 0.0 & $2.9 \mathrm{D}-13$ & 4.0 & $2.4 \mathrm{D}-12$ & 2.0 \\
\hline 0.75 & 0.757 & $1.3 \mathrm{D}-14$ & - & 0.0 & $1.2 \mathrm{D}-12$ & 4.0 & $4.8 \mathrm{D}-12$ & 2.0 \\
\hline 1.5 & 1.515 & $2.1 \mathrm{D}-13$ & 16.7 & 0.0 & $4.7 \mathrm{D}-12$ & 4.0 & $9.8 \mathrm{D}-12$ & 2.0 \\
\hline 3 & 3.035 & $3.5 \mathrm{D}-12$ & 16.5 & 0.0 & $1.9 \mathrm{D}-11$ & 4.0 & $2.0 \mathrm{D}-11$ & 2.1 \\
\hline 6 & 6.092 & $6.0 \mathrm{D}-11$ & 16.9 & 0.0 & $7.6 \mathrm{D}-11$ & 4.0 & $4.5 \mathrm{D}-11$ & 2.2 \\
\hline 12 & 12.274 & $9.9 \mathrm{D}-10$ & 16.6 & 0.0 & $3.0 \mathrm{D}-10$ & 3.9 & $1.0 \mathrm{D}-10$ & 2.3 \\
\hline 24 & 24.941 & $1.4 \mathrm{D}-8$ & 13.9 & 0.0 & $9.9 \mathrm{D}-10$ & 3.3 & $2.3 \mathrm{D}-10$ & 2.3 \\
\hline 48 & 51.721 & $1.2 \mathrm{D}-7$ & 8.6 & 0.0 & $2.3 \mathrm{D}-9$ & 2.3 & $4.3 \mathrm{D}-10$ & 1.8 \\
\hline
\end{tabular}


О ТОЧНОСТИ АППРОКСИМАЦИИ ДВИЖЕНИЯ МАЛОГО НЕБЕСНОГО ТЕЛА

\begin{tabular}{|c|c|c|c|c|c|c|c|c|c|c|}
\hline \multirow{7}{*}{ 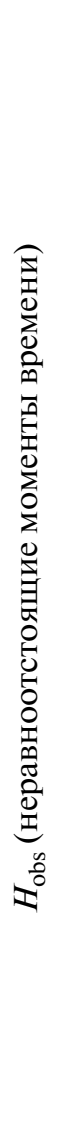 } & 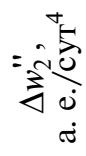 & $\begin{array}{l}\overrightarrow{1} \\
\stackrel{1}{\partial} \\
\stackrel{+}{+}\end{array}$ & $\begin{array}{l}\overline{1} \\
1 \\
n \\
\infty \\
\infty\end{array}$ & $\begin{array}{l}\stackrel{0}{1} \\
\stackrel{1}{g} \\
\stackrel{1}{i}\end{array}$ & $\begin{array}{l}\stackrel{0}{1} \\
\stackrel{1}{\rho} \\
\stackrel{\theta}{\theta}\end{array}$ & $\begin{array}{l}\stackrel{0}{1} \\
\stackrel{1}{a} \\
\hat{\sigma}\end{array}$ & $\stackrel{q}{\stackrel{i}{q}}$ & $\begin{array}{l}\hat{1} \\
\hat{a} \\
\hat{i}\end{array}$ & సิ & $\begin{array}{l}\infty \\
1 \\
n \\
n \\
n\end{array}$ \\
\hline & 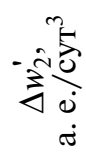 & $\begin{array}{c}\stackrel{1}{1} \\
\stackrel{1}{\ominus} \\
\dot{n}\end{array}$ & $\begin{array}{l}\stackrel{\sim}{1} \\
\stackrel{1}{\theta} \\
\stackrel{\theta}{r}\end{array}$ & $\begin{array}{l}\overrightarrow{1} \\
\stackrel{1}{a} \\
\vec{i}\end{array}$ & $\begin{array}{l}\exists \\
1 \\
\stackrel{1}{a} \\
\infty\end{array}$ & $\begin{array}{l}\stackrel{0}{1} \\
\stackrel{1}{a} \\
\text { ஸे }\end{array}$ & 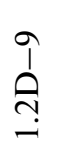 & 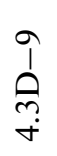 & $\begin{array}{l}\infty \\
1 \\
\stackrel{1}{*}\end{array}$ & $\begin{array}{l}\hat{I} \\
\text { fे } \\
\text { o }\end{array}$ \\
\hline & 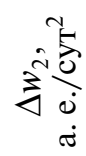 & $\begin{array}{l}\bar{\jmath} \\
\hat{\jmath} \\
\hat{\sigma}\end{array}$ & $\begin{array}{l}\frac{1}{1} \\
\stackrel{1}{i} \\
\stackrel{2}{i}\end{array}$ & $\begin{array}{l}\sim \\
1 \\
\infty \\
\infty \\
\dot{m}\end{array}$ & $\begin{array}{l}\overline{7} \\
1 \\
\dot{b} \\
\dot{n}\end{array}$ & $\begin{array}{l}\stackrel{0}{1} \\
\stackrel{1}{0} \\
\stackrel{n}{+}\end{array}$ & 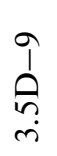 & $\begin{array}{l}\infty \\
1 \\
\infty \\
\substack{0 \\
i}\end{array}$ & în & $\stackrel{n}{1}$ \\
\hline & 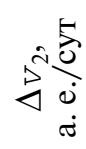 & $\begin{array}{l}\hat{i} \\
\hat{a} \\
a\end{array}$ & $\begin{array}{l}0 \\
1 \\
\rho \\
\infty \\
\dot{m}\end{array}$ & $\begin{array}{l}\stackrel{0}{1} \\
1 \\
\hat{n} \\
\text { n̊ }\end{array}$ & 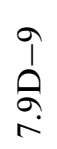 & 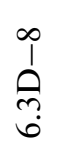 & $\begin{array}{l}\hat{1} \\
\hat{\theta} \\
\text { in }\end{array}$ & $\begin{array}{l}0 \\
1 \\
O \\
\dot{+}\end{array}$ & $\begin{array}{l}n \\
n \\
n \\
n \\
n\end{array}$ & $\stackrel{?}{1}$ \\
\hline & $\dot{\sim} \dot{\forall} \dot{\sim}$ & $\begin{array}{l}\hat{I} \\
\hat{a} \\
\text { in }\end{array}$ & $\stackrel{i}{i}$ & $\begin{array}{l}\infty \\
1 \\
\underline{.}\end{array}$ & $\stackrel{i}{\text { I̦ }}$ & $\begin{array}{l}0 \\
1 \\
\text { m. }\end{array}$ & î & $\begin{array}{l}\hat{1} \\
\hat{a} \\
\infty \\
\infty\end{array}$ & 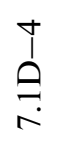 & $\begin{array}{l}\hat{I} \\
\text { nิ } \\
\text { nn }\end{array}$ \\
\hline & 穵态 & $\begin{array}{l}\stackrel{0}{1} \\
\stackrel{1}{0} \\
\hat{n}\end{array}$ & $\stackrel{0}{\circ}$ & $\stackrel{0}{0}$ & $\begin{array}{l}\stackrel{0}{1} \\
\stackrel{1}{m} \\
\stackrel{\rho}{i}\end{array}$ & 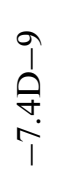 & $\begin{array}{l}\infty \\
1 \\
\infty \\
\infty \\
i \\
1\end{array}$ & $\begin{array}{l}\hat{1} \\
\hat{0} \\
\dot{+}\end{array}$ & $\begin{array}{l}0 \\
1 \\
\varrho \\
\dot{+}\end{array}$ & $\begin{array}{l}+ \\
\stackrel{+}{1} \\
\stackrel{i}{i}\end{array}$ \\
\hline & $\stackrel{3}{1}$ & $\begin{array}{l}\frac{n}{2} \\
\hat{o} \\
0 \\
0\end{array}$ & $\frac{n}{\frac{n}{\infty}}$ & 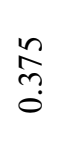 & $\stackrel{n}{0}$ & $\cong$ & $m$ & 6 & $\simeq$ & $\underset{\sim}{ \pm}$ \\
\hline \multirow{7}{*}{ 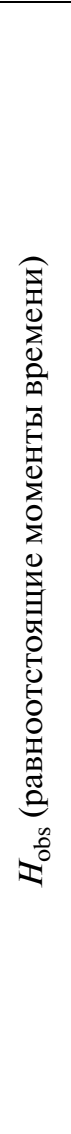 } & 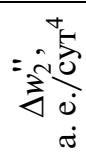 & $\begin{array}{l}\stackrel{\nabla}{1} \\
\stackrel{1}{0} \\
\dot{\theta}\end{array}$ & $\begin{array}{l}n \\
\stackrel{1}{1} \\
\stackrel{0}{0} \\
\stackrel{1}{1}\end{array}$ & $\begin{array}{l}n \\
1 \\
n \\
n \\
a\end{array}$ & $\begin{array}{l}\stackrel{1}{1} \\
\stackrel{1}{\infty} \\
\dot{m}\end{array}$ & $\begin{array}{l}\overrightarrow{7} \\
\stackrel{1}{n} \\
n\end{array}$ & 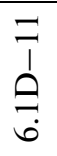 & $\begin{array}{l}\stackrel{0}{1} \\
1 \\
\stackrel{\partial}{\partial} \\
i\end{array}$ & 市 & $\begin{array}{l}\infty \\
1 \\
\text { } \\
\end{array}$ \\
\hline & 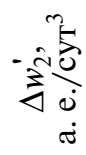 & $\begin{array}{l}\mathfrak{d} \\
\stackrel{1}{a} \\
\stackrel{\sim}{\sim}\end{array}$ & $\begin{array}{l}\exists \\
\stackrel{1}{\prime} \\
\exists\end{array}$ & $\begin{array}{l}\vec{J} \\
\stackrel{1}{\vartheta} \\
\stackrel{+}{+}\end{array}$ & $\begin{array}{l}\stackrel{0}{1} \\
1 \\
\stackrel{1}{2}\end{array}$ & $\begin{array}{l}0 \\
1 \\
\rho \\
0 \\
0\end{array}$ & $\stackrel{\hat{i}}{\hat{i}}$ & $\begin{array}{l}\infty \\
1 \\
ٍ \\
ٍ\end{array}$ & 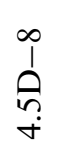 & İ \\
\hline & 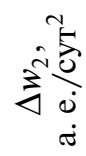 & $\begin{array}{l}\stackrel{1}{1} \\
\stackrel{1}{0} \\
\infty\end{array}$ & $\begin{array}{l}\overline{1} \\
\stackrel{1}{\rho} \\
\stackrel{0}{0}\end{array}$ & $\begin{array}{l}\stackrel{1}{1} \\
\stackrel{1}{\sim}\end{array}$ & $\begin{array}{l}m \\
\stackrel{1}{1} \\
\mathfrak{\infty}_{0}\end{array}$ & 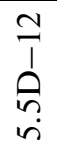 & $\begin{array}{l}\overline{1} \\
\hat{1} \\
\hat{a} \\
\infty\end{array}$ & م̂ & 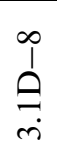 & $\begin{array}{l}0 \\
1 \\
i \\
i\end{array}$ \\
\hline & 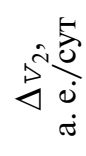 & 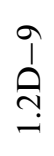 & $\stackrel{i}{\stackrel{i}{f}}$ & $\begin{array}{l}0 \\
1 \\
\stackrel{1}{\theta}\end{array}$ & $\begin{array}{l}\stackrel{0}{1} \\
\stackrel{1}{\rho} \\
\stackrel{-}{-}\end{array}$ & $\begin{array}{l}\stackrel{0}{1} \\
1 \\
\stackrel{1}{n}\end{array}$ & $\begin{array}{l}\infty \\
1 \\
ٍ \\
ٍ\end{array}$ & $\stackrel{\hat{I}}{\hat{a}}$ & $\begin{array}{l}0 \\
1 \\
\rho \\
\dot{\sigma}\end{array}$ & $\stackrel{+}{\stackrel{+}{1}}$ \\
\hline & $\begin{array}{l}\dot{\sim} 0 \\
\dot{\forall} \dot{\sigma}\end{array}$ & 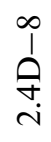 & $\begin{array}{l}\infty \\
1 \\
\infty \\
\infty \\
i\end{array}$ & $\frac{\hat{1}}{n}$ & $\stackrel{i}{\hat{1}}$ & $\begin{array}{l}\infty \\
1 \\
0 \\
0 \\
0\end{array}$ & $\begin{array}{l}i \\
i n \\
i n \\
i\end{array}$ & $\begin{array}{l}\dot{p} \\
\text { in } \\
\text { mे } \\
\dot{*}\end{array}$ & $\begin{array}{l}n \\
1 \\
\infty \\
\infty \\
\infty\end{array}$ & $\begin{array}{l}n \\
\hat{n} \\
\end{array}$ \\
\hline & సิ & $\stackrel{0}{\circ}$ & $\stackrel{0}{\circ}$ & $\stackrel{0}{0}$ & $\stackrel{0}{0}$ & $\stackrel{0}{0}$ & $\stackrel{i}{i}$ & $\begin{array}{l}\infty \\
1 \\
n \\
n \\
i\end{array}$ & $\begin{array}{l}\hat{I} \\
\hat{\theta} \\
\dot{i n}\end{array}$ & $\stackrel{n}{\hat{i}}$ \\
\hline & $\stackrel{5}{\stackrel{5}{5}}$ & $\stackrel{2}{\stackrel{2}{0}}$ & $\stackrel{\imath}{3}$ & $\tilde{0}$ & - & $N$ & $\nabla$ & $\infty$ & $\underline{\sigma}$ & กี \\
\hline
\end{tabular}




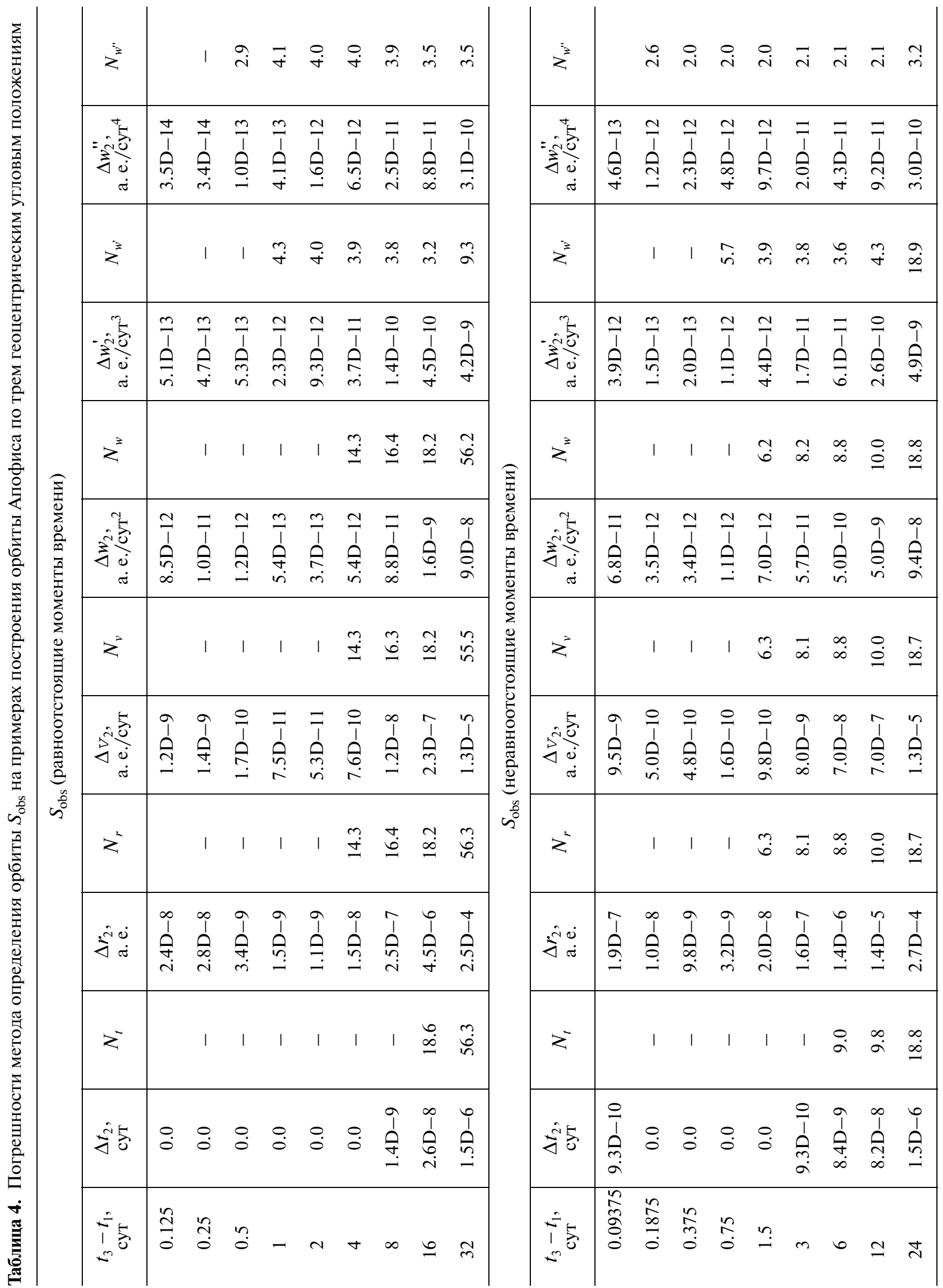


число $n . m \times 10^{-k}$ ( $n, m$ и $k-$ целые числа). Длина опорного интервала $\left(t_{3}-t_{1}\right)$ уменьшалась в два раза до тех пор, пока ограничения на представление чисел в компьютере не приводили к заметному ухудшению точности вычислений. Прочерк в таблицах означает, что отношение значений ошибок методов в данном случае не могло быть вычислено корректно из-за того, что ошибки округления оказались сопоставимыми по величине с методическими погрешностями.

Полученные результаты показывают, что сокращение опорного интервала $\left(t_{3}-t_{1}\right)$ (при малых его размерах) в два раза приводит к уменьшению погрешностей сравниваемых методов приблизительно в $2^{N}$ раз $(N=1,2,3,4)$, что полностью согласуется с Утверждениями 1 и 2 . Из сравнения погрешностей построения орбит $H_{\mathrm{pos}}$ и $H_{\mathrm{obs}}$ с погрешностями построения орбит $S_{\mathrm{pos}}$ и $S_{\mathrm{obs}}$ соответственно видно, что для самых коротких опорных интервалов времени (до 0.5 сут) точности аппроксимации реального движения по основным параметрам (координатам и скоростям), достигнутые с помощью сравниваемых методов, практически одинаковы. В этих случаях, по указанным выше причинам, результаты вычислений перенасыщены ошибками округлений, и сравнение методов представляется недостаточно корректным. С увеличением опорного интервала времени [при $\left(t_{3}-t_{1}\right)>0.5$ сут] методы построения орбит $H_{\text {pos }}$ И $H_{\text {obs }}$ проигрывают в точности методам построения орбит $S_{\text {pos }}$ и $S_{\text {obs }}$ соответственно. Этот проигрыш по данным последних строк таблиц достигает 1.5-2 порядков. Основное объяснение этому состоит в том, что формулы построения орбит $H_{\text {pos }}$ и $H_{\text {obs }}$ опираются на разложения решений в ряды Тейлора, в которых отброшены члены пятого и более высоких порядков. Предложенные же в S07 и S10 методы основаны на замкнутых (без разложения в ряды) выражениях, которые позволяют полностью учесть эффекты кеплеровской составляющей движения (1) и основную часть возмущающего ускорения.

\section{ЗАКЛЮЧЕНИЕ}

В данной работе рассмотрены предложенные ранее первым автором методы определения орбиты по трем векторам положения и трем наблюдениям, позволяющие учесть основную часть возмущений в движении исследуемого малого небесного тела. Теоретически исследован вопрос о сходимости решений, получаемых с помощью этих методов, к точным решениям при сокращении опорного интервала времени, определяемого крайними моментами наблюдений. Исследование показало, что орбиты, которые строятся с помощью указанных методов, на 1-2 порядка точнее, чем орбиты, изученные нами в предыдущей работе (SS15). По результатам исследования сформулированы два утверждения. Ранее рассмотренные и новые численные примеры подтверждают справедливость полученных законов изменения погрешностей методов.

Исследование первого автора в рамках данной работы выполнено по заданию № 2014/223 (код проекта 1567) Министерства образования и науки Российской Федерации. Исследование второго автора выполнено по заданию № 645 (код проекта 4.1349.2014) Министерства образования и науки Российской Федерации.

\section{СПИСОК ЛИТЕРАТУРЫ}

Шефер B.A. Сверхоскулирующие промежуточные орбиты: касания четвертого и пятого порядков к траекториям возмущенного движения // Письма в Астрон. журн. 2006. Т. 32. № 12. С. 937-951.

Шефер В.А. Определение промежуточной возмущенной орбиты по трем векторам положения // Письма в Астрон. журн. 2007. Т. 33. № 4. С. 307-320.

Шефер B.A. Новый метод определения промежуточной орбиты по трем положениям малого тела на небесной сфере // Астрон. вестн. 2010. Т. 44. № 2. С. $167-$ 181. (Shefer V.A. A new method for the determination of an intermediate orbit using three positions of a small body on the celestial sphere // Sol. Syst. Res. 2010. V. 44. №. 2. P. $152-165$.)

Шефер B.A., Шефер O.B. О точности аппроксимации движения малого небесного тела промежуточными возмущенными орбитами, построенными по двум векторам положения и трем наблюдениям // Астрон. вестн. 2015. Т. 49. № 1. C. 54-64. (Shefer V.A., Shefer O.V. On the accuracy of approximation of a small celestial body motion using intermediate perturbed orbits calculated from two position vectors and three observations // Sol. Syst. Res. 2015. V. 49. № 1. P. 51-60.)

Herrick S. Jupiter IX and Jupiter XII // Publ. Astron. Soc. Pac. 1952. V. 64. P. 237-241. 